\title{
A Sensing System for Simultaneous Detection of Urine and its Components Using Plastic Optical Fibers
}

\author{
Tahseen Ejaz* Non-member \\ Tadashi Takemae* Non-member \\ Chikara Egami* Non-member \\ Naoyuki Tsuboi* Student Member
}

\begin{abstract}
A sensing system using plastic optical fibers and reagent papers was developed for the detection of urine and abnormal level of its components simultaneously. Among several components of urine the detection of two main components namely, protein and glucose was confirmed experimentally. Three states of the papers namely dry and wet with and without change in color, were taken into consideration. These three states were divided by setting the lower and upper threshold voltages at $2.2 \mathrm{~V}$ and $5.5 \mathrm{~V}$, respectively. This system is considered to be simple in construction, easy to operate and cost-efficient.
\end{abstract}

Keywords : urinary incontinence, bedsore, sensing system, plastic optical fibers, reagent papers

\section{Introduction}

Urinary incontinence is the inability to control urination. People who suffer from urinary incontinence can't hold their urine - they wet themselves. Incontinence is a serious problem for patients with disabilities and those who are terminally ill. These patients are usually made to wear diapers that are changed at certain intervals in order to prevent them from wetting their bed. However, if the patient urinates in between the intervals and remains unattended, wearing the wet diaper for a long time can cause bedsore. Therefore, it is important to change the diaper as soon as it is wet rather than at certain intervals. In order to detect urinary incontinence, some sensors have been developed ${ }^{(1) \sim(3)}$. However, these are either complex in design or cost much to maintain.

Poor health condition is considered to be another reason for bedsore. In clinics, health conditions of patients are determined by urine test using reagent papers that can distinguish between normal and abnormal urine. In this paper, a simple and cheap sensing system for the simultaneous detection of urinary incontinence and abnormal level of its components using plastic optical fibers and reagent papers is introduced. As an example of the detection of components, the sensing of two main components of urine namely, protein and glucose was performed experimentally.

\section{Principle of Operation}

The transmittance of light passing through a dry paper is much lower than that through a wet paper. This principle is used in the detection of urinary incontinence. In the presence of abnormal level of protein and glucose, the colors of corresponding reagent papers change from light yellow to dark green and from light green to dark brown, respectively. The transmittance of light passing through a wet light-colored reagent paper is higher than that through a wet dark-colored paper. Converting the transmittance of light into voltage, a time-voltage characteristics

* Shizuoka University Faculty of Engineering 3-5-1 Johoku, Hamamatsu 432-8561

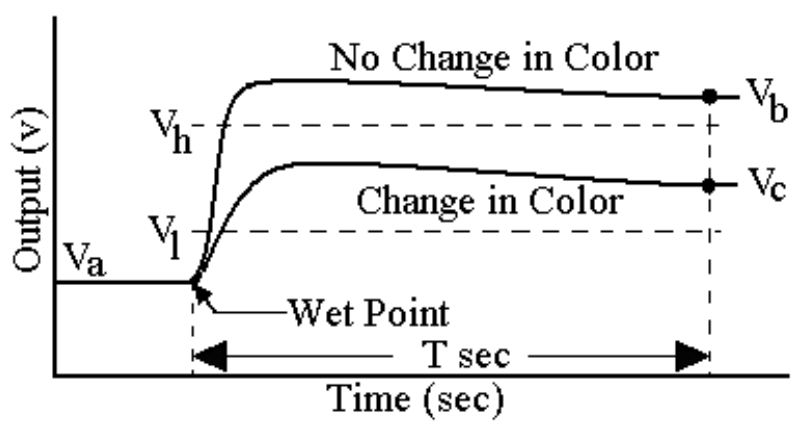

Fig. 1. The predicted time-voltage characteristics

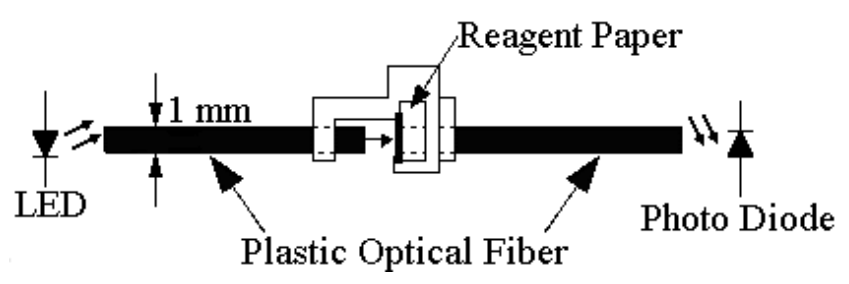

Fig. 2. The sensor part

under three different circumstances as shown in Fig. 1 can be predicted. In this figure, $\mathrm{V}_{\mathrm{a}}$ is the output voltage when the paper is dry. When the paper gets wet at the Wet Point without any change in color, the voltage rises above the upper threshold voltage $V_{h}$ and reaches a steady value $V_{b}$ after $T$ seconds. However, if there is a change in the color after the paper is wet, the output voltage reaches a steady value $V_{c}$ after $T$ seconds that is above the lower threshold voltage $V_{1}$ but below $V_{h}$. These three different levels of voltages under three different circumstances, i.e. dry, wet without change in color and wet with change in color can be used to detect the urinary incontinence and the abnormal level of its components.

\section{The Sensing System}

The sensor part of the sensing system for each component 


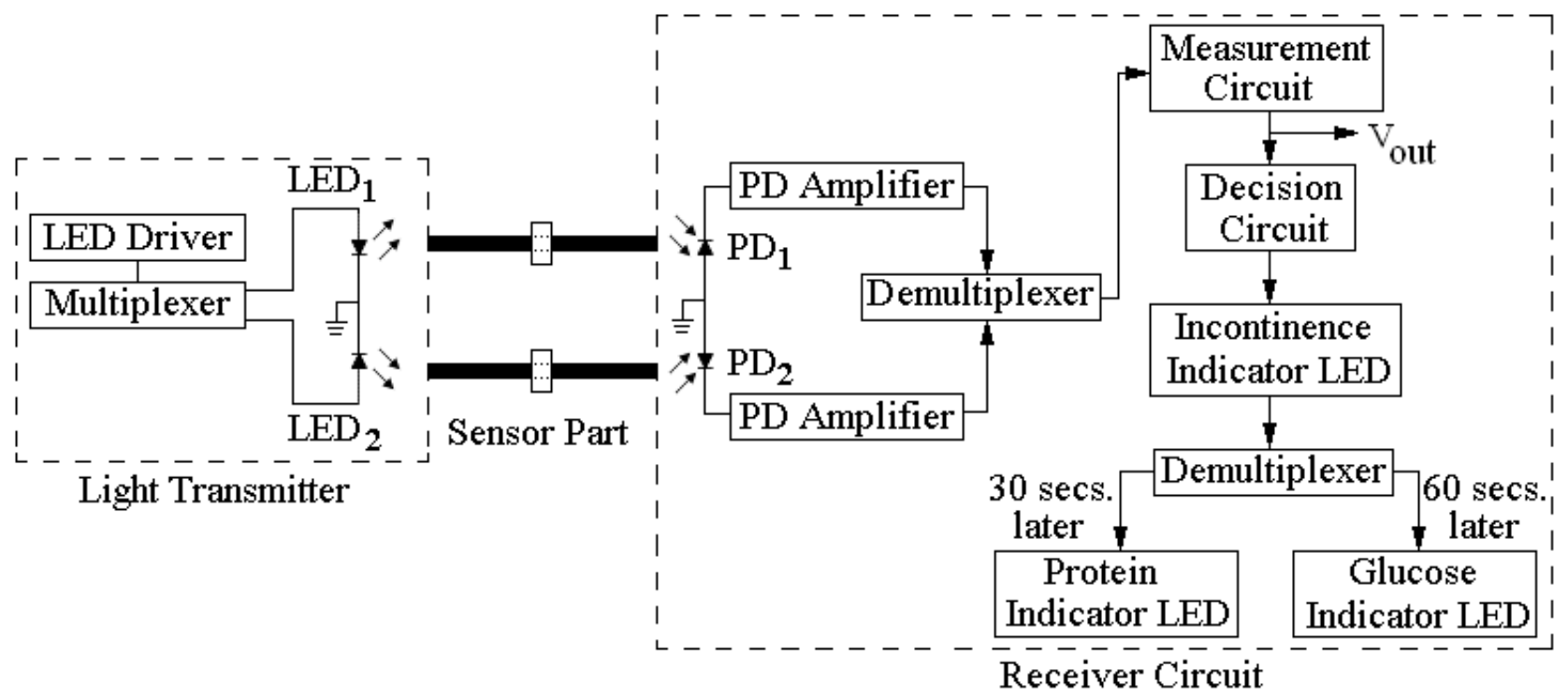

Fig. 3. The block diagram

consists of a reagent paper for the respective component and two plastic optical fibers $1 \mathrm{~mm}$ in diameter as shown in Fig. 2. The paper is kept in a small plastic holder. There is an LED at one end of the fibers that faces the top surface of the reagent paper and acts as light transmitter. A photodiode at the other end acts as receiver.

The block diagram of the sensing system is shown in Fig. 3. It consists of three parts: light transmitter, sensor part and receiver circuit. A square wave of $500 \mathrm{~Hz}$ is used as input signal for the light emitter in order to avoid interference from other light sources. The receiver circuit comprises of an analog circuit for measuring the voltage and a digital circuit for making decision about the status of the signal. Two LEDs are used as light transmitters in the system. One of the LEDs indicates incontinence and abnormal level of protein; the other indicates abnormal level of glucose. The timing sequence of the circuit is controlled by multiplexers in such a way that the sensing of the components is done one at a time for a period of 30 seconds each. This time is required for the color of the reagent paper to be changed in the presence of abnormal levels of urinary components.

\section{Experiments and Results}

At first, the appropriate colors of light transmitter LEDs for each component were selected. This was done by measuring the output voltage $\mathrm{V}_{\text {out }}$ using different colored LEDs under three different conditions, namely dry, wet with and without change in color. From this data, red and orange colored LEDs of wavelength $610 \mathrm{~nm}$ and $700 \mathrm{~nm}$ were chosen for the sensing of protein and glucose, respectively that showed significant difference in voltages between these conditions. The graph for the LED test is shown in Fig. 4. Using this graph, the lower and upper threshold voltages $\mathrm{V}_{1}$ and $\mathrm{V}_{\mathrm{h}}$ were set at $2.2 \mathrm{~V}$ and $5.5 \mathrm{~V}$, respectively.

In order to detect urinary incontinence and protein, a dry yellow colored reagent paper was kept into the plastic holder of the sensor part and the output voltage $\mathrm{V}_{\text {out }}$ was measured under the three different circumstances. The time-voltage waveform as obtained from the experiment is shown in Fig. 5. $\mathrm{V}_{\text {out }}$ measured with dry paper remained below $\mathrm{V}_{1}$ due to the poor transmittance of light passing through the paper. The paper was then soaked into water

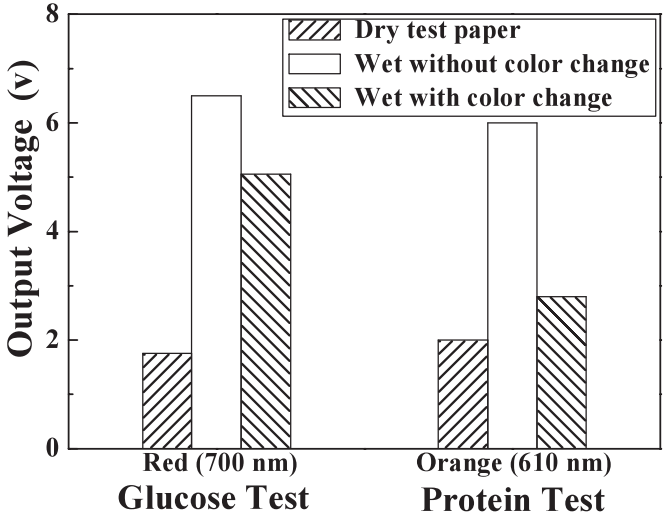

Fig. 4. Data of LED test for protein and glucose

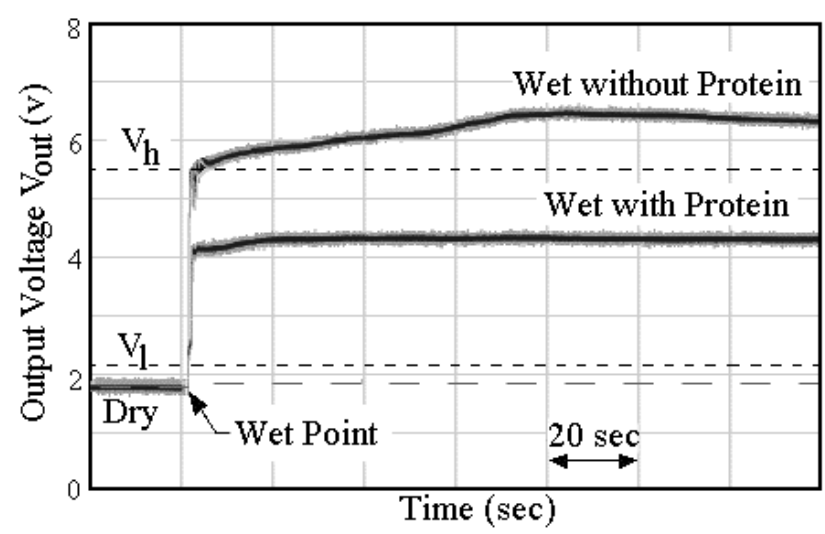

Fig. 5. Waveform as obtained from protein test

substituted for normal urine at the wet point. The increase in transmittance caused $V_{\text {out }}$ to rise above $V_{h}$ and reach a steady value. The incontinence indicator LED was lit as soon as $V_{\text {out }}$ rose above $\mathrm{V}_{1}$ but 30 seconds later the protein indicator LED was not lit. Another dry yellow colored reagent paper was soaked into water 


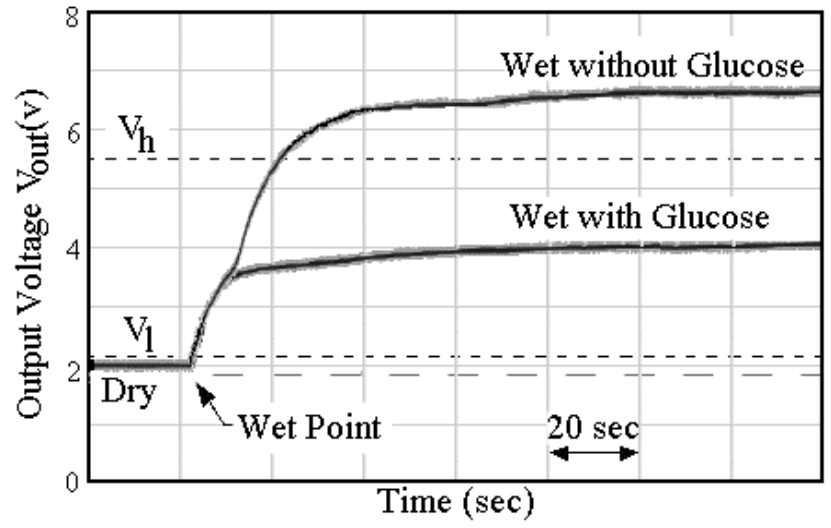

Fig. 6. Waveform as obtained from glucose test

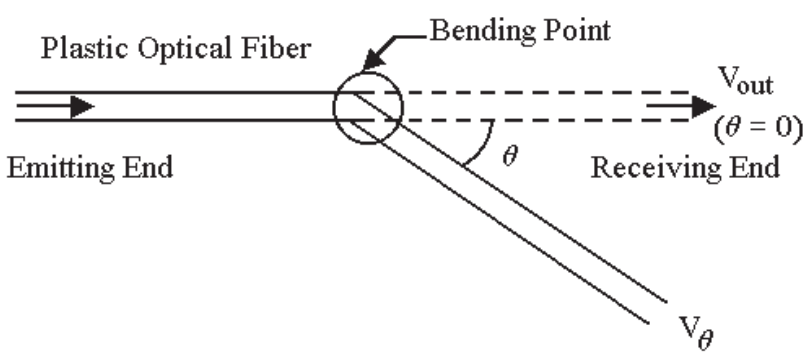

(i)

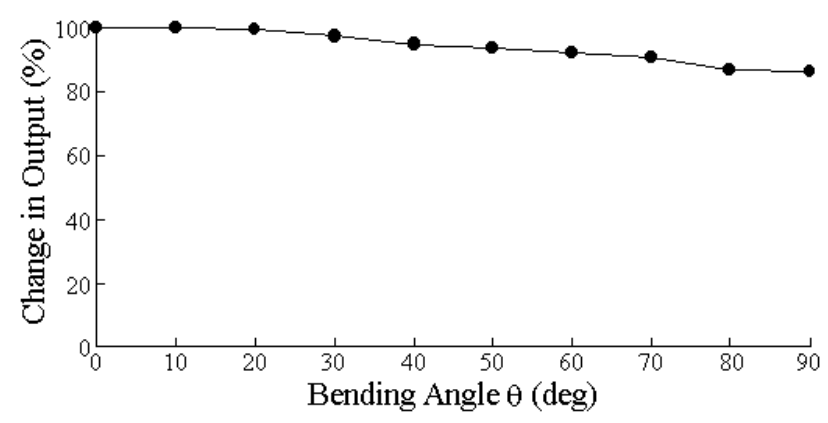

(ii)

Fig. 7. (i) Bending of the plastic optical fiber and (ii) experimental data showing changes in output with bending angle

mixed with protein that was substituted for urine with protein and the change in $\mathrm{V}_{\text {out }}$ was observed. The color of the paper changed into green within 30 seconds resulting in lower transmittance. $V_{\text {out }}$ rose above $V_{1}$ but remained below $V_{h}$ and reached a steady value 30 seconds later. In this case, both the incontinence indicator LED and protein indicator LED were lit. For the detection of glucose, a similar procedure as above was followed with light green colored reagent paper. The experimental results are shown in Fig. 6.

In order to check whether the bending of the plastic optical fiber affects the output or not, $\mathrm{V}_{\text {out }}$ was measured while varying the bending angle $\theta$ of the fiber from $0^{\circ}$ to $90^{\circ}$ in $10^{\circ}$ steps as shown in Fig. 7(i). The ratio of the output voltages at different values of $\theta$ and at $\theta=0^{\circ}$ were calculated. The results obtained from this test are shown in Fig. 7(ii).

\section{Discussion}

In order to provide better health care for bed ridden patients it is necessary to detect abnormality in urine along with urinary incontinence. For this purpose, a new sensing system using plastic optical fibers was proposed for the sensing of protein and glucose and its operation was confirmed from the results of the experiments. The results from the protein and glucose tests indicate that the output voltage is higher for plain water than that for water mixed with protein and glucose. From Fig. 4 it is evident that the normal and abnormal states of urine can be clearly divided by selecting two threshold voltages. Since the fiber is only $1 \mathrm{~mm}$ in diameter, the wetness of the spot of the reagent paper in front of the fiber is sufficient enough to increase the output voltage $V_{\text {out }}$ above the upper threshold voltage $\mathrm{V}_{\mathrm{h}}$ for normal urine irrespective of the volume of urine.

The results as shown in Fig. 7(ii) indicate that the bending of the fibers has very little effect on the output voltage, thereby making the proper functioning of the sensor much less vulnerable to the movement of the patient. Since the sensor part consists only of plastic optical fibers, a plastic holder and reagent paper, and the only replaceable part is the paper, the system is very economical.

\section{Conclusion}

In this paper, a new sensing system for the simultaneous detection of urine and its components was proposed. The detection of protein and glucose was confirmed experimentally. Other components can also be detected by increasing the number of light transmitter LEDs, sensor parts and component indicator LEDs and by readjusting the timing sequence of the multiplexer. The detection of urinary components is considered to be one of the methods of checking the condition of health of the patients. Using the results of the detection, better health-care can be provided for the patients, namely, by controlling the diet. The flexible nature and small diameter $(1 \mathrm{~mm})$ of plastic optical fibers makes the sensor part of the system suitable for use in diapers. In hospitals, the sensing system can be used with telemeters to send data of various patients to a computer for centralized observation.

(Manuscript received Nov. 1, 2002. revised March 6, 2003)

\section{References}

(1) E. D. James, F. C. Flack, K. P. S. Caldwell, and M. R. Martin : "Continuous measurement in urine loss and frequency in incontinent patients", $\mathrm{Br} . \mathrm{J}$. Urol., Vol.43, pp.233-237 (1971)

(2) S. L. Stanton and D. Ritchie : "Urilos: The practical detection of urine loss", Am. J. Obstet. Gynecol., Vol.128, pp.461-463 (1977)

(3) K. Nakajima, T. Tamura, H. Miike, S. Shimooki, T. Fujimoto, and T. Nakano : "Disposable diaper with urinary incontinence monitor", Japanese J. Med., Elect. Bio. Engg., 32-2, pp.15-23 (1994)

Tahseen Ejaz

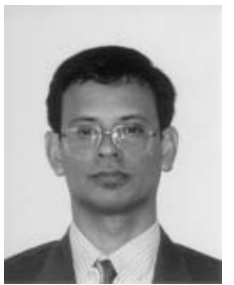

(Non-member) He Received Ph.D in electronic engineering from the Shizuoka University Faculty of Engineering in 2001. Worked as post doctorate researcher at the Venture Business Laboratory of the university until March 2003. Currently working as a researcher at the Shizuoka University Joint Research Center. His research interests are biomedical engineering, electronic sensing and image processing. 
Tadashi Takemae

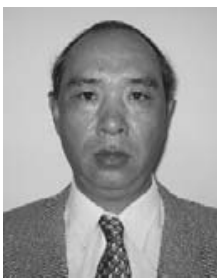

(Non-member) was born in Nagano, Japan, on September9, 1952. He received a Ph.D. degree in electronic engineering from the Tokyo Institute of Technology in 1991, and presently a Professor at the Faculty of Engineering, Shizuoka University. His current research interests are simulation and measurement of biological substances.

Chikara Egami

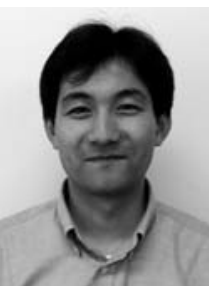

(Non-member) was born in Hokkaido, Japan, on November 6, 1965. He received a ph.D degree in applied physics from Muroran Institute of Technology in 1993, and is presently an associate professor at Shizuoka Universtiy. He has worked on nonlinear optics, and development of optical memory. Japan Applied Physics Society, Optical Society of America member.
Naoyuki Tsuboi

(Student Member) was born in Shizuoka, Japan, on June 20,1979 . He received a master's degree in electronic engineering from Shizuoka University in 2003, and is presently working in Panasonic Mobile Communications Shizuoka R\&D Lab.Co.,Ltd. He is currently working on the development of mobile phone. 